PROCEEDINGS OF THE

AMERICAN MATHEMATICAL SOCIETY

Volume 130, Number 3, Pages $773-777$

S 0002-9939(01)06179-2

Article electronically published on August 28, 2001

\title{
THE LARGEST LINEAR SPACE OF OPERATORS SATISFYING THE DAUGAVET EQUATION IN $L_{1}$
}

\author{
R. V. SHVYDKOY
}

(Communicated by Dale Alspach)

\begin{abstract}
We find the largest linear space of bounded linear operators on $L_{1}(\Omega)$ that, being restricted to any $L_{1}(A), A \subset \Omega$, satisfy the Daugavet equation.
\end{abstract}

\section{INTRODUCTION}

Let $(\Omega, \Sigma, \mu)$ be an arbitrary measure space without atoms of infinite measure. Also let $\Sigma^{+}=\{A \in \Sigma: \mu(A)>0\}$. If $A \in \Sigma^{+}, L_{1}(A)$ stands for the space of (classes of) real-valued $\mu$-integrable functions supported on $A$. If $T$ is a bounded linear operator on $L_{1}(\Omega)$ and $A \in \Sigma^{+}$, we denote by $T_{A}$ the restriction of $T$ onto $L_{1}(A)$. Finally, $\mathcal{L}\left(L_{1}(\Omega)\right)$ denotes the space of all bounded linear operators on $L_{1}(\Omega)$.

The purpose of this note is to give an explicit description of the largest linear space $\mathcal{M}$ of operators $T \in \mathcal{L}\left(L_{1}(\Omega)\right)$ satisfying the following identity:

$$
\left\|I d_{A}+T_{A}\right\|=1+\left\|T_{A}\right\|
$$

for any set $A \in \Sigma^{+}$.

Originally, (1) was established by Daugavet for compact operators on $C[0,1]$ (see [2]). The case of $L_{1}$ was first treated by Lozanovskii in his paper [6], where he proved Daugavet's theorem for compact operators in $L_{1}[0,1]$ (see also [1]). Later, Holub generalized this result for all weakly compact operators on an arbitrary atomless $L_{1}(\Omega)$ (see 4]). Plichko and Popov in their work [7 found a still broader (in case of atomless $\mu$ ) linear class of so-called narrow operators satisfying the Daugavet equation, and in fact their proof works for operators from $L_{1}(A)$ to $L_{1}(\Omega)$, whenever $A \in \Sigma^{+}$.

So, finding the largest class of such operators naturally completes this line of results.

We also refer the reader to papers [5] and 8] for recent developments and applications of the Daugavet theory.

Received by the editors November 12, 1999 and, in revised form, September 15, 2000.

2000 Mathematics Subject Classification. Primary 47B38; Secondary 46E30.

Key words and phrases. Daugavet equation, weakly compact operators, narrow operators.

The author wishes to thank V. M. Kadets for stimulating discussions and useful remarks.

(C)2001 American Mathematical Society 


\section{MAIN RESULT}

In the sequel it is convenient to denote $\Sigma_{A}^{+}=\left\{B: B \subset A, B \in \Sigma^{+}\right\}$, whenever $A \in \Sigma^{+}$.

We define $\mathcal{M}$ as the set of all operators $T \in \mathcal{L}\left(L_{1}(\Omega)\right)$ that meet the following condition:

$$
\begin{aligned}
& \text { For every } \varepsilon>0 \text { and } A \in \Sigma^{+} \\
& \text {there is a } B \in \Sigma_{A}^{+} \text {with } \mu(B)<\infty \text { such that } \\
& \left\|\chi_{B} \cdot T\left(\frac{\chi_{B}}{\mu(B)}\right)\right\|<\varepsilon .
\end{aligned}
$$

This condition simply means that the operator $T$ can shift sufficiently many functions from their supports.

Let us state our main result.

Theorem 1. Every linear space of operators satisfying (11) for any $A \in \Sigma^{+}$is contained in $\mathcal{M}$, and $\mathcal{M}$ itself is a closed linear space consisting of such operators.

The main ingredient in the proof of this theorem is the following proposition.

Proposition 2. For an operator $T \in \mathcal{L}\left(L_{1}(\Omega)\right)$ the following conditions are equivalent:

(i) $T$ and $-T$ satisfy (11) for all $A \in \Sigma^{+}$;

(ii) for every $\varepsilon>0$ and $A \in \Sigma^{+}$there is an $A^{\prime} \in \Sigma_{A}^{+}$such that if $B \in \Sigma_{A^{\prime}}^{+}$, then we can find a $B^{\prime} \in \Sigma_{B}^{+}$with the following properties:
a) $\left\|\frac{\chi_{B^{\prime}}}{\mu\left(B^{\prime}\right)}-\frac{\chi_{B}}{\mu(B)}\right\|<\varepsilon$,
b) $\left\|\chi_{B^{\prime}} \cdot T\left(\frac{\chi_{B^{\prime}}}{\mu\left(B^{\prime}\right)}\right)\right\|<\varepsilon$;

(iii) $T \in \mathcal{M}$.

Proof. (i) implies (ii). We begin with the following observation.

Suppose $S: L_{1}(A) \mapsto L_{1}(\Omega)$ is a bounded linear operator. Then for any given $\varepsilon>0$ there is a set $A_{1} \in \Sigma_{A}^{+}$with $\mu\left(A_{1}\right)<\infty$ such that for every non-negative function $f \in S\left(L_{1}\left(A_{1}\right)\right)$ we have $\|S f\| \geq\|S\|-\varepsilon$.

Indeed, we can assume that $\mu(A)<\infty$ and choose $g^{*} \in S\left(L_{1}^{*}(\Omega)\right)$ so that $\left\|S^{*} g^{*}\right\|>\|S\|-\varepsilon$. Then, regarding $S^{*} g^{*}$ as an element of $L_{\infty}(A)$ we find a set $A_{1} \in \Sigma_{A}^{+}$with $\theta S^{*} g^{*}\left(A_{1}\right) \subset(\|S\|-\varepsilon,\|S\|]$, where $\theta$ is a sign. Now, if $f \in S\left(L_{1}(A)\right)$, $f \geq 0$ and $\operatorname{supp}(f) \subset A_{1}$, then $\|S f\|>\theta g^{*}(S f)=\theta S^{*} g^{*}(f)>\|S\|-\varepsilon$, from where the observation follows.

We know that $\left\|I d_{A}+T_{A}\right\|=1+\left\|T_{A}\right\|$. By scaling, without loss of generality we can and do assume that $\left\|T_{A}\right\|=1$. So there is an $A_{1} \in \Sigma_{A}^{+}$with $\mu\left(A_{1}\right)<\infty$ such that

$$
\left\|\frac{\chi_{B}}{\mu(B)}+T\left(\frac{\chi_{B}}{\mu(B)}\right)\right\|>2-\varepsilon
$$

whenever $B \in \Sigma_{A_{1}}^{+}$. We also know that $\left\|I d_{A_{1}}-T_{A_{1}}\right\|=1+\left\|T_{A_{1}}\right\|>2-\varepsilon$. Thus there exists an $A^{\prime} \in \Sigma_{A_{1}}^{+}$such that

$$
\left\|\frac{\chi_{B}}{\mu(B)}-T\left(\frac{\chi_{B}}{\mu(B)}\right)\right\|>2-\varepsilon,
$$

whenever $B \in \Sigma_{A^{\prime}}^{+}$. 
We prove that $A^{\prime}$ is the desired set.

To this end, let us fix $B \in \Sigma_{A^{\prime}}^{+}$. It follows from (3), (44) and a theorem of Dor [3] that there are two disjoint measurable sets $\Omega_{1}$ and $\Omega_{2}$ in $\Omega$ such that

$$
\int_{\Omega_{1}}\left|T\left(\frac{\chi_{B}}{\mu(B)}\right)\right|(t) d \mu(t)>(1-\varepsilon)^{2}
$$

and

$$
\int_{\Omega_{2}} \frac{\chi_{B}}{\mu(B)}(t) d \mu(t)>(1-\varepsilon)^{2} .
$$

The last inequality implies

$$
\begin{aligned}
\mu\left(B \cap \Omega_{1}\right) & =\mu(B) \int_{B \cap \Omega_{1}} \frac{\chi_{B}}{\mu(B)}(t) d \mu(t)<\mu(B) \int_{\Omega \backslash \Omega_{2}} \frac{\chi_{B}}{\mu(B)}(t) d \mu(t) \\
& <\left(1-(1-\varepsilon)^{2}\right) \mu(B)=\left(2 \varepsilon-\varepsilon^{2}\right) \mu(B) .
\end{aligned}
$$

Let us put $B^{\prime}=B \backslash \Omega_{1}$ and show that $B^{\prime}$ meets conditions a) and b).

First,

$$
\begin{aligned}
\left\|\frac{\chi_{B^{\prime}}}{\mu\left(B^{\prime}\right)}-\frac{\chi_{B}}{\mu(B)}\right\| & =\int_{\Omega}\left|\frac{\chi_{B^{\prime}}}{\mu\left(B^{\prime}\right)}-\frac{\chi_{B^{\prime}}}{\mu(B)}+\frac{\chi_{B^{\prime}}}{\mu(B)}-\frac{\chi_{B}}{\mu(B)}\right|(t) d \mu(t) \\
& \leq 1-\frac{\mu\left(B^{\prime}\right)}{\mu(B)}+\frac{\mu\left(B \cap \Omega_{1}\right)}{\mu(B)}=2 \frac{\mu\left(B \cap \Omega_{1}\right)}{\mu(B)}
\end{aligned}
$$

and taking into account (6), we obtain

$$
\left\|\frac{\chi_{B^{\prime}}}{\mu\left(B^{\prime}\right)}-\frac{\chi_{B}}{\mu(B)}\right\|<2\left(2 \varepsilon-\varepsilon^{2}\right) .
$$

Second, from (5), (7) and $\left\|T_{A}\right\|=1$ it follows that

$$
\begin{aligned}
\left\|\chi_{B^{\prime}} \cdot T\left(\frac{\chi_{B^{\prime}}}{\mu\left(B^{\prime}\right)}\right)\right\| & =\int_{B^{\prime}}\left|T\left(\frac{\chi_{B^{\prime}}}{\mu\left(B^{\prime}\right)}\right)\right|(t) d \mu(t) \\
& <\int_{B^{\prime}}\left|T\left(\frac{\chi_{B}}{\mu(B)}\right)\right|(t) d \mu(t)+2\left(2 \varepsilon-\varepsilon^{2}\right) \\
& \leq \int_{\Omega \backslash \Omega_{1}}\left|T\left(\frac{\chi_{B}}{\mu(B)}\right)\right|(t) d \mu(t)+2\left(2 \varepsilon-\varepsilon^{2}\right) \\
& \leq 3\left(2 \varepsilon-\varepsilon^{2}\right) .
\end{aligned}
$$

In view of the arbitrariness of $\varepsilon$, this gives the desired result.

It is obvious that (iii) follows from (ii).

Let us finally prove that (iii) implies (i). Since $\mathcal{M}$ is stable under scalar multiplication, it is sufficient to prove (1) only for $T$.

To this end, we fix an arbitrary $A \in \Sigma^{+}$and as above for any given $\varepsilon>0$ we find an $A^{\prime} \in \Sigma_{A}^{+}$with $\mu\left(A^{\prime}\right)<\infty$ such that for every $B \in \Sigma_{A^{\prime}}^{+},\left\|T\left(\frac{\chi_{B}}{\mu(B)}\right)\right\|>\left\|T_{A}\right\|-\varepsilon$. By condition (21), there is a $B_{0} \in \Sigma_{A^{\prime}}^{+}$such that $\left\|\chi_{B_{0}} \cdot T\left(\frac{\chi_{B_{0}}}{\mu\left(B_{0}\right)}\right)\right\|<\varepsilon$. This means that $\frac{\chi_{B_{0}}}{\mu\left(B_{0}\right)}$ and $T\left(\frac{\chi_{B_{0}}}{\mu\left(B_{0}\right)}\right)$ are almost disjoint functions, and as a consequence we 
have the following estimate:

$$
\begin{aligned}
\left\|I d_{A}+T_{A}\right\| \geq & \left\|\frac{\chi_{B_{0}}}{\mu\left(B_{0}\right)}+T\left(\frac{\chi_{B_{0}}}{\mu\left(B_{0}\right)}\right)\right\| \\
= & \int_{B_{0}}\left|\frac{\chi_{B_{0}}}{\mu\left(B_{0}\right)}+T\left(\frac{\chi_{B_{0}}}{\mu\left(B_{0}\right)}\right)\right|(t) d \mu(t)+\int_{\Omega}\left|T\left(\frac{\chi_{B_{0}}}{\mu\left(B_{0}\right)}\right)\right|(t) d \mu(t) \\
& -\int_{B_{0}}\left|T\left(\frac{\chi_{B_{0}}}{\mu\left(B_{0}\right)}\right)\right|(t) d \mu(t) \\
> & 1-\varepsilon+\left\|T_{A}\right\|-\varepsilon-\varepsilon=1+\left\|T_{A}\right\|-3 \varepsilon .
\end{aligned}
$$

This finishes the proof.

Now we are in a position to prove our main result.

Proof of Theorem 11. Proposition 2 implies that $\mathcal{M}$ consists of operators satisfying (11) for all $A \in \Sigma^{+}$, and that every linear space of such operators is contained in $\mathcal{M}$. $\mathcal{M}$ is obviously closed and stable under scaling. So, the only thing we have to prove is that if operators $U$ and $V$ belong to $\mathcal{M}$, then their sum belongs to $\mathcal{M}$ too. To show this, we check condition (ii) of Proposition 2 for $U+V$. Further on, we assume that $\|V\| \leq 1$.

Indeed, let $A \in \Sigma^{+}$and $\varepsilon>0$ be arbitrary. Applying Proposition 2 to the operator $U$ we find a set $A^{\prime} \in \Sigma_{A}^{+}$as in condition (ii). Then, by the same proposition applied to $V$ we find a set $A^{\prime \prime} \in \Sigma_{A^{\prime}}^{+}$with the corresponding properties. To show that $A^{\prime \prime}$ is the required set, suppose $B \in \Sigma_{A^{\prime \prime}}^{+}$. By the choice of $A^{\prime \prime}$ there is a $B^{\prime} \in \Sigma_{B}^{+}$such that

$$
\left\|\frac{\chi_{B^{\prime}}}{\mu\left(B^{\prime}\right)}-\frac{\chi_{B}}{\mu(B)}\right\|<\frac{\varepsilon}{4}
$$

and

$$
\left\|\chi_{B^{\prime}} \cdot V\left(\frac{\chi_{B^{\prime}}}{\mu\left(B^{\prime}\right)}\right)\right\|<\frac{\varepsilon}{4} .
$$

Since $B^{\prime} \subset A^{\prime}$, by the analogous property of $A^{\prime}$, there is a $B^{\prime \prime} \in \Sigma_{B^{\prime}}^{+}$with

$$
\left\|\frac{\chi_{B^{\prime \prime}}}{\mu\left(B^{\prime \prime}\right)}-\frac{\chi_{B^{\prime}}}{\mu\left(B^{\prime}\right)}\right\|<\frac{\varepsilon}{4}
$$

and

$$
\left\|\chi_{B^{\prime \prime}} \cdot U\left(\frac{\chi_{B^{\prime \prime}}}{\mu\left(B^{\prime \prime}\right)}\right)\right\|<\frac{\varepsilon}{2} .
$$

From (8) and (10) we get $\left\|\frac{\chi_{B^{\prime \prime}}}{\mu\left(B^{\prime \prime}\right)}-\frac{\chi_{B}}{\mu(B)}\right\|<\varepsilon$. So, if we prove that $\left\|\chi_{B^{\prime \prime}} \cdot V\left(\frac{\chi_{B^{\prime \prime}}}{\mu\left(B^{\prime \prime}\right)}\right)\right\|$ $<\frac{\varepsilon}{2}$, then $\left\|\chi_{B^{\prime \prime}} \cdot(V+U)\left(\frac{\chi_{B^{\prime \prime}}}{\mu\left(B^{\prime \prime}\right)}\right)\right\|<\varepsilon$, and we are done. But this easily follows from (9), (10) and the facts that $\|V\| \leq 1$ and $B^{\prime \prime} \subset B^{\prime}$.

The proof is completed.

\section{REFERENCES}

[1] V. F. Babenko and S. A. Pichugov, On a property of compact operators in the space of integrable functions, Ukranian Math. J. 33 (1981), 374-376. MR 82m:47018

[2] I. K. Daugavet, On a property of completely continuous operators in the space C, Uspekhi Mat. Nauk, 18.5 (1963), 157-158 (Russian). MR 28:461

[3] L. E. Dor, On projections in $L_{1}$, Ann. of Math. 102 (1975), 463-474. MR 54:8258 
[4] J. R. Holub, Daugavet's equation and operators on $L_{1}(\mu)$, Proc. Amer. Math. Soc. 100 (1987), 295-300. MR 88j:47037

[5] V. M. Kadets, R. V. Shvidkoy, G. G. Sirotkin, and D. Werner, Banach spaces with the Daugavet property, Trans. Amer. Math. Soc. 352, N.2 (2000), 855-873. MR 2000c:46023

[6] G. Y. Lozanovsky, On almost integral operators in KB-spaces, Vestnik Leningrad. Univ., 7 (1966), 35-44.

[7] A. M. Plichko and M. M. Popov, Symmetric function spaces on atomless probability spaces, Dissertationes. Mat. 306 (1990), 1-85. MR 92f:46032

[8] R. V. Shvidkoy, Geometric aspects of the Daugavet property, J. Funct. Anal. 176 (2000), 198-212. CMP 2001:01

Department of Mathematics, University of Missouri - Columbia, Columbia, Missouri 65211

E-mail address: shvidkoy@math.missouri.edu 